\title{
The Death of a Young Adult due to Wallenberg Syndrome: A Case Report
}

Ariyarathna HTDW*

Department of Forensic Medicine, Faculty of Medical Sciences, University of Sri Jayewardenepura. Sri Lanka.

\begin{abstract}
Wallenberg syndrome which is also known as lateral medullary syndrome (LMS) and posterior inferior cerebellar artery syndrome ((PICA syndrome) is detected relatively rarely among young adults. A 42-year-old apparently healthy male presented with headache, vomiting and vertigo. He was diagnosed to have severe hypertension and type-2 diabetes mellitus. During his first admission his first non-contrast computed tomography (NCCT) scan of the brain had confirmed a cerebellar infarction. With clinical findings, the patient was treated as a possible case of LMS. With the repeat NCCT on the third day, he was diagnosed to have progressive cerebellar infarction and a medullary infarction. In the following day the patient was discharged with reserved dates for vertebral artery duplex and ultrasound scan of abdomen (USS). On the seventh day of the illness he had collapsed and died. Subsequent autopsy revealed a left-sided cerebellar and a brain stem infarction along with generalized cerebral oedema. Important findings deduced by forensic pathologists should be conveyed to the clinicians in order to broaden the treatment options and to prevent premature deaths.
\end{abstract}

Keywords: Cerebellar infarction, Lateral Medullary Syndrome (LMS), neuroimaging, Posterior Inferior Cerebellar Artery Syndrome (PICA syndrome), Wallenberg syndrome

Received: 10 May 2021, Revised version accepted: 26 June 2021, Published: 30 June 2021. *Corresponding author: Ariyarathna HTDW, $\triangle$ Email: ariyaratna@sjp.ac.lk (D) https://orcid.org/0000-0002-4266-5508

Cite this article as: Ariyarathna HTDW. The Death of a Young Adult due to Wallenberg Syndrome: A Case Report. MedicoLegal Journal of Sri Lanka, 2021;9(1):28-32. DOI: http://doi.org/10.4038/mljsl.v9i1.7426

Copyright: @ 2019 with the Medico-legal Journal of Sri Lanka.

This is an open-access article distributed under the terms of the Creative Commons Attribution 4.0 International License, which permits unrestricted use, distribution, and reproduction in any medium provided the original author and source are credited.

\section{Introduction}

The clinical importance of cerebellar infarctions is considerable because of the life threatening postinfarction brain stem compression by a postinfarction oedema. The triad of Horner's syndrome, ipsilateral limb ataxia, and contralateral limb numbness reliably indicated the involvement of vascular territory of Posterior Inferior Cerebellar Artery (PICA) resulting in Wallenberg syndrome/LMS. A secondary oedema, herniation, obstructive hydrocephalus, a secondary infarction or a propagation of a thrombus are also to be considered in this young patient especially because of the progressive infarction confirmed with a CT scan at the time of discharge. A magnetic resonance imaging (MRI) of brain would have perhaps saved his life.[1,2] The option of "treatability" of a given death is an important concept to broaden the management options.[3]

\section{Case report}

A 42-year-old male, who was in apparent good health presented himself to hospital with a sudden onset of headache, vertigo and vomiting. On the second day of admission, he had experienced a left hemi-facial sensory loss, a right upper and lower limb sensory loss with dysphagia, dysarthria, and left sided cerebellar signs. In his medical history he was diagnosed for the first time to have diabetes mellitus and hypertension. The first Non Contrast Computed (NCCT) Tomography, brain of the patient showed a left cerebellar infarction and with the second NCCT brain it had progressed to the lateral medulla as well (Fig. 1 A \& B). The 2D echo had revealed mild concentric hypertrophy of the left ventricle and had excluded possible cardiac thromboembolism. The dysphagia, deviation of the uvula to the right, left-sided cerebellar signs and leftsided Horner's syndrome, horizontal nystagmus also been elicited. By the fourth day, the patient had been discharged along with a nasogastric (NG) tube because of his dysphagia with the plan of being subjected to vertebral artery duplex and an ultrasound scan abdomen to exclude any renal pathology causing secondary hypertension and with a plan of physiotherapy. On the day three after being discharged (almost 7 days after the initial symptoms) in the morning he had indicated that he wanted to defecate and had mentioned that he felt unwell and had suddenly collapsed and died. Upon the inquest, a postmortem examination was performed. No (add internal or external) features of trauma were evident. He died before the due date of the vertebral artery duplex scan. 
The brain was oedematous(weight $1400 \mathrm{~g}$ and the dura was tensed) with no midline shift and there was a marked swelling over the left cerebellar hemisphere with macroscopical area of infarction measuring $3.5 \times 4 \mathrm{~cm}$ in size mostly involving the inferior surface and also the superior surface. There was no gray and white matter demarcations and the area showed necrotic brain matter (Fig. 4). No brain stem haemorrhages were noted but left -sided tonsillar herniation was noticed (Fig. 3 A \& B). The circle of Willis appeared anatomically normal with patchy atherosclerosis mainly in the fourth part of the left vertebral artery, bilateral PICA, proximal basilar artery, left posterior cerebral artery, and in an anterior cerebral artery a with longitudinal dissection (no cross sections were studied). The left PICA showed a haemorrhagic area on the atheromatous plaque (Fig. 1). The heart was 320 grams in weight with no ischaemic changes. Microscopically the cerebellar infarctions with neutrophil infiltrations were seen with marked oedema (Fig. 5 A, B, C \& D). There were no autopsy findings to suggest deep vein thrombosis or pulmonary thromboembolism.

The cause of death was determined as cerebral oedema with herniation due to the left cerebellar infarction with PICA syndrome with the probable underlying cause of severe atherosclerotic lesions of the circle of Willis due to uncontrolled hypertension and diabetes mellitus.
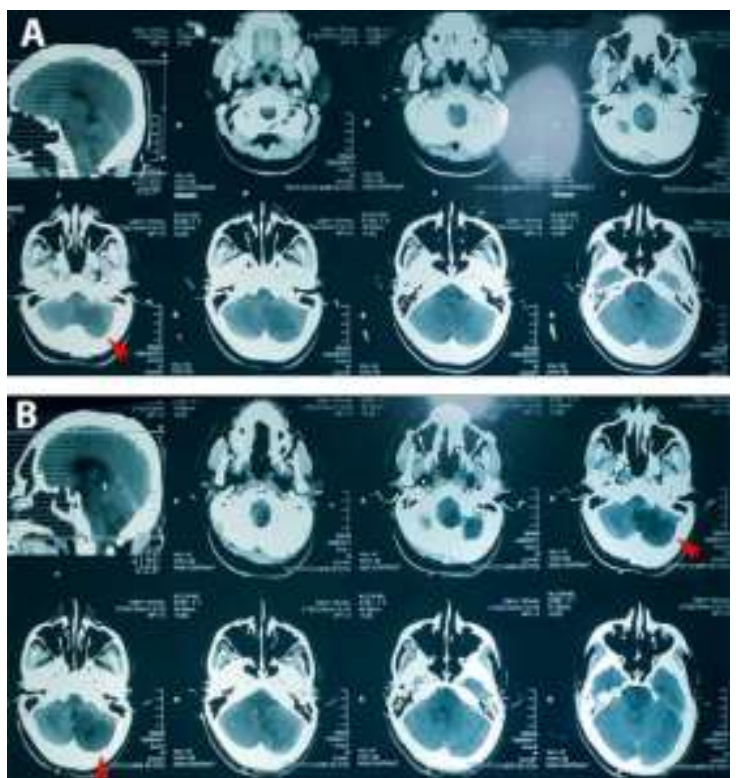

Figure 1. (A) The NCCT brain performed on the first day of admission. (B) The NCCT brain showing progressive infarctions in the day 3(indicated with arrows).

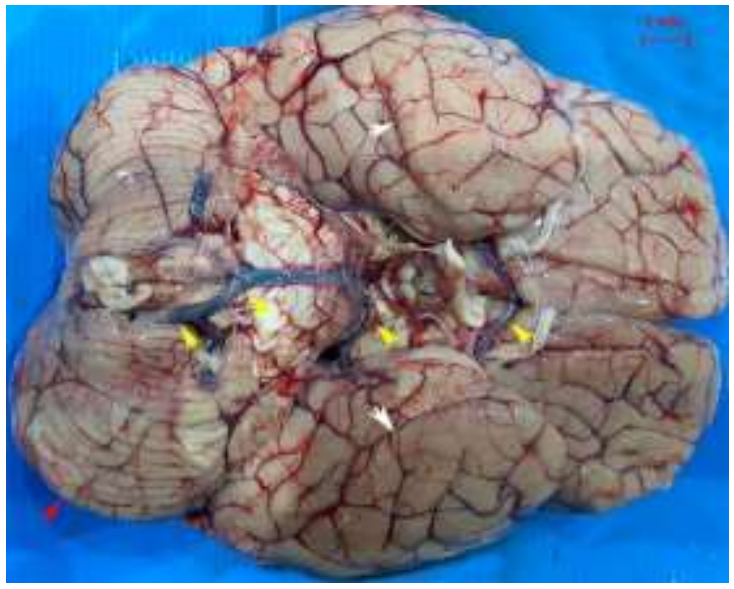

Figure 2. The photograph of the base of the brain with the oedematous and enlarged left cerebellar hemisphere (red arrow head) and the thickened arterial walls of the circle of Willis (marked with yellow arrow heads). Abnormal bilateral indentations of the basal temporal lobes caused by the upward pressure exerted by the space-occupying lesion of the posterior compartment against the falxcerebri (white arrow heads).
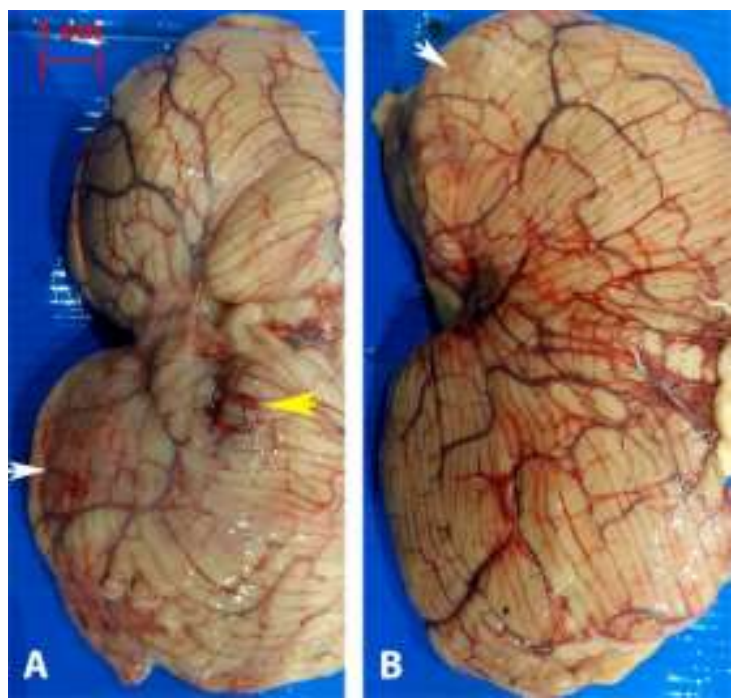

Figure 3. (A) A photograph of the infarcted left tonsils and oedematous and congested left inferior cerebellar hemisphere (arrow head). (B) The superior surface of the cerebellum with extensive left- sided necrosis (arrow head). 


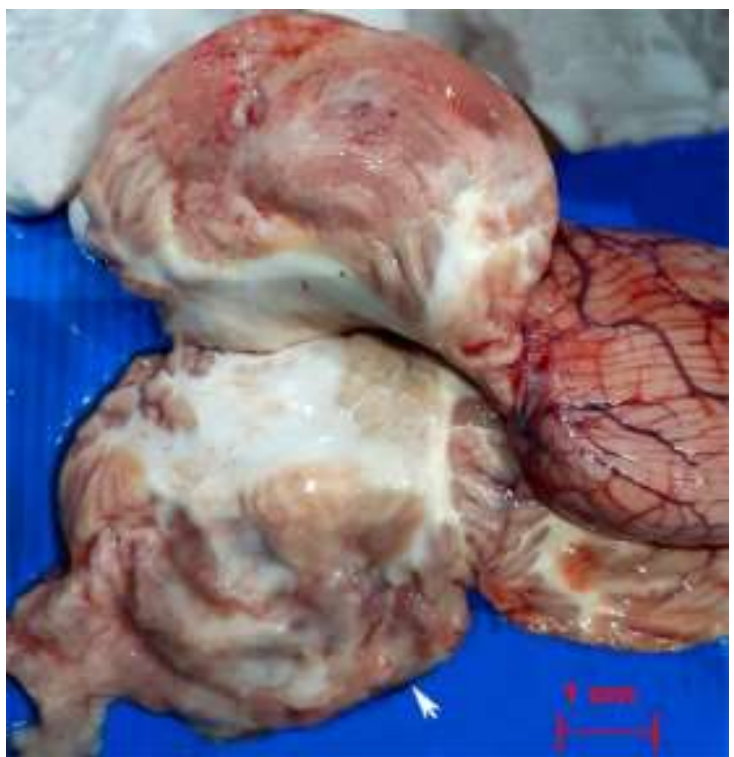

Figure 4. A photograph of the transverse section of the cerebellum illustrating the ischemic necrosis of the left cerebellar hemisphere (the white arrow head).
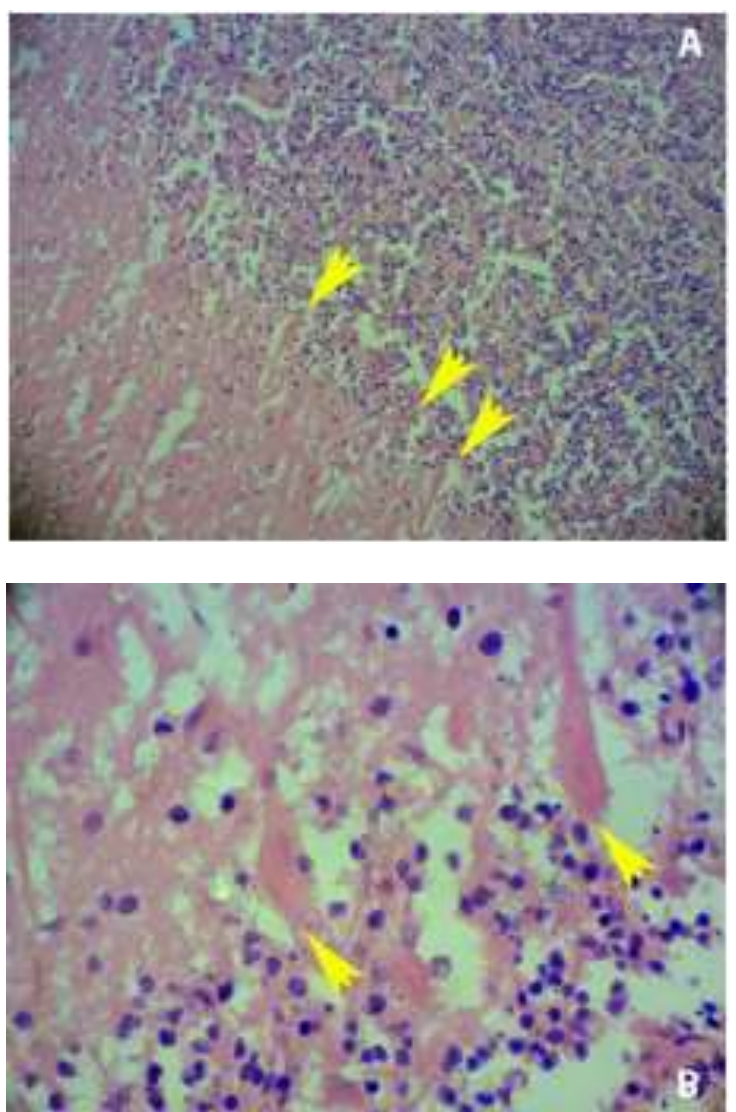

Figure 5. (H\&E A x 4 and B x 40). The cerebellar neurons are reduced to barely visible outlines with no or hardly seen nuclei inside, (ghost cells, marked with yellow arrows).
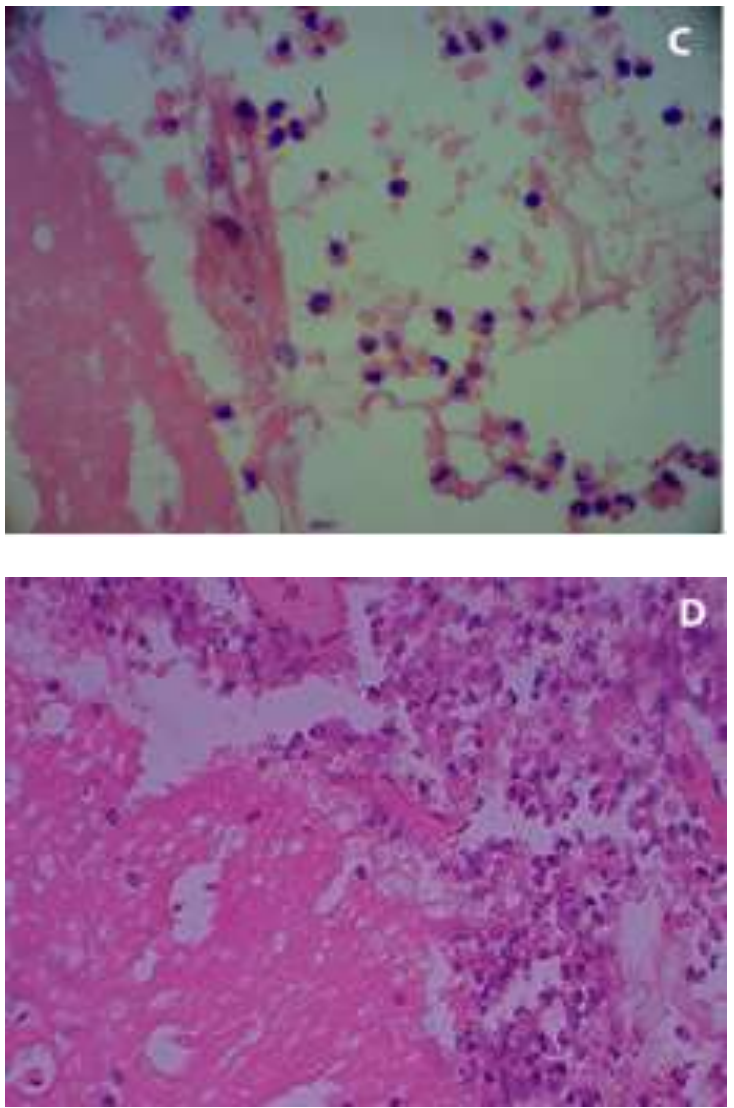

Figure 5. (H\&E stain, $x 10 \mathrm{C}$ and 4 D) Numerous Polymorphonuclear leukocytes are visible diffusely within the parenchyma of the left cerebellar hemisphere).

\section{Discussion}

In the purview of management of cerebrovascular Accident (CVA), brain neuroimaging plays an essential role as it helps to distinguish the salvageable tissues from the already irreversibly damaged brain tissues, to identify vascular malformations, to plan the treatment options such as intravenous thrombolysis or intra-arterial thrombectomy and also to predict the outcomes.[4] Since the infarcted area was progressive as confirmed by the second NCCT brain, an MRI scan before being discharged would have been a right decision in order to consider the treatment options such as in finding of an occluded artery and then to have treatment options such as thrombolysis and also would have been able to pinpoint the extent of the infarction.[5] At the time of discharge the infarction was significantly extensive (Figure 1). The known causes of thrombotic phenomenon in the vasculature of the brain in descending order of frequency are due to atherosclerosis, thromboembolism, vasculopathies including arterial dissection especially at vertebral arteries, embolization of venous thrombosis, and right to left shunt.[6] In this deceased, no history of congenital heart diseases, any coagulopathies, or any trauma was evident. Evidence of chronic arterial disease 
was present and the wall of the fourth part of the left vertebral was thickened with atherosclerosis and the lumen was not occluded at the postmortem though there had been a few places with haemorrhag eruptured plaques (but no image is there to illustrate it). The thickened wall was seen up to the proximal left basilar artery. The left PICA was also dilated and there were mild atherosclerosis when compared to the right PICA (Figure 2).

In this case "wet cutting" of the brain was adopted though the brain along with the circle of Willis should have been formalin fixed before sectioning in order to obtain thinner and more accurate sections for a better histological preservation. Furthermore, it could have led to a complete picture if the histology of circle of Willis was done along with a control sample from the periphery of the body in order to exclude any pathology of arterial walls with special stains as well. Another drawback of this case study is the fact that the author was unable to come up with any new remarkable finding as to what caused the unexpected death other than what the clinicians had already diagnosed.

Though the LMS is not a fatal disease in this patient's case, it turned out fatal, as it was associated with a significant cerebellar infarction. [7] The most important concern, in this case, was the probability of saving his life. According to the literature review, PICA/LMS is a disease in the older age group and it averages the 6th decade [8] of the life but even in that age group death is not expected when it is presented in a non-complicated manner.[7] The possibility of a second CVA causing cerebral oedema cannot be excluded in this case. Or else the extension of the necrosis or ischemia due to the propagation of a thrombus was also quite possible. The dating of the incident or onset of the disease, is unable to be done precisely in this case but it can be mentioned as a recent event.[9] The available histopathological sections were evident for polymorpho-nuclear neutrophils within the necrotic cerebellum. The relative scarcity of Purkinje neurons and the presence of barely visible neuronal outlines indicated a recent infarction (Figures 5.A \& B).[10] But there were no red neurons (acidophilic neurons) to suggest an acute infarction occurred within 12-24 hours.[9]

Forensic pathologists encounter cases where the concept of "treatability" is applicable as in this case. It is recommended to have a mechanism to convey the postmortem findings within the same hospital as it will invariably serve to broaden the management outcomes of the clinicians.

\section{Conclusions}

PICA syndrome/LMS can even occur among young adults. Undiagnosed hypertension and diabetes mellitus were the two contributory causes of this patient that were recognized. The significantly large cerebellar infarction that was progressive could have been considered before merely discharging the patient and the concerns of "treatability" are existent in this case. Nevertheless, a propagation of the thrombus or a secondary infarction was unable to be excluded.

\section{Acknowledgements}

Dr. Isha Premathilaka (Senior Lecturer/Consultant Histopathologist, Department of Pathology, University of Sri Jayewardenepura (USJ) for providing necessary information regarding histopathology. For micro-photographing Mr MSE Premalal (Technical officer) from the Department of Pathology and Ms. M.W Sooriyarchchi, Ms WMCW Menike, Ms NGH Kawushalya from the Department of Parasitology, USJ, Dr. A. Kiruththihan for the neurology opinion (Registrar in Medicine, Colombo South Teaching Hospital), Mrs SC Kaluarchchi (Technical Officer), EMMS Ekanayaka, ADA Vimukthi, JAN Kawshalya, RADLD Ranawaka, AI Rangika and KDS Chandimal from the Department of Forensic Medicine, USJ for preparation of histology slides and for the miscellaneous support and Mr. Wasantha Manawadu for the IT assistance (Technical Officer, Dean's office, USJ).

\section{Disclosure statement}

Conflict of interest: The author declares that there is no conflict of interest.

Funding: None

\section{References}

1. Edlow JA, Newman-Toker DE, Savitz SI. Diagnosis and initial management of cerebellar infarction. Lancet Neurol 2008;7(10):951-64. DOI: 10.1016/S1474-4422(08)70216-3

2. Cocker LJLD, Lövblad K-O, Hendrikse J. MRI of Cerebellar Infarction. Eur Neurol. 2017;77(34):137-46. DOI: $10.1159 / 000455229$

3. OECD iLibrary. Avoidable-mortality2019.Joint-OECD-Eurostat. -List-preventabletreatable-causes-of-death. [cited 2020 Oct 29]. Available from: https://www.oecd.org/health/ health-systems/Avoidable-mortality-2019-JointOECD-Eurostat-List-preventable-treatablecauses-of-death.pdf

4. Hand PJ, Kwan J, Lindley RI, Dennis MS, Wardlaw JM. Distinguishing between stroke and mimic at the bedside: the brain attack study. Stroke. 2006; 37(3):769-75. DOI: 10.1161/01.STR.0000204041.13466.4c

5. Shafaat O, Sotoudeh H. Stroke Imaging. In: StatPearls. Treasure Island (FL): StatPearls Publishing; 2020 [cited 2020 Oct 29]. Available from: http:/www.ncbi.nlm.nih.gov/books/NBK $546635 /$ 
6. Wallenberg Syndrome. Physiopedia. [cited 2020 Oct 28]. Available from: https://www.physiopedia.com/Wallenberg_Syndrome.

7. Lateral Medullary Syndrome - an overview. ScienceDirect Topics. [cited 2020 Oct 29]. Available from: https://www.sciencedirect.com/ topics/neuroscience/lateral-medullary-syndrome

8. Kozak HH, Uca AU, Poyraz N, Anliaçık SÖ, Tokgöz OS. Clinical and radiologic features and their relationships with neurofunctional scores in patients with acute cerebellar infarct. Ann Indian Acad Neurol. 2016;19(2):211-5. DOI: 10.4103/0972-2327.177351

9. Garman RH. Histology of the Central Nervous System. Toxicol Pathol. 2011 Jan 1;39(1):22-35. DOI: $10.1177 / 0192623310389621$

10. Rahaman P, Del Bigio M. Histology of Brain Trauma and Hypoxia-Ischemia. Academic Forensic Pathology. 2018;8(3):539-54. doi: $10.1177 / 1925362118797728$ 